\title{
Timelike and Spacelike Matter Inheritance Vectors in Specific Forms of Energy-Momentum Tensor
}

\author{
M. Sharif *and Umber Sheikh \\ Department of Mathematics, University of the Punjab, \\ Quaid-e-Azam Campus Lahore-54590, PAKISTAN.
}

\begin{abstract}
This paper is devoted to the investigation of the consequences of timelike and spacelike matter inheritance vectors in specific forms of energy-momentum tensor, i.e., for string cosmology (string cloud and string fluid) and perfect fluid. Necessary and sufficient conditions are developed for a spacetime with string cosmology and perfect fluid to admit a timelike matter inheritance vector, parallel to $u^{a}$ and spacelike matter inheritance vector, parallel to $x^{a}$. We compare the outcome with the conditions of conformal Killing vectors. This comparison provides us the conditions for the existence of matter inheritance vector when it is also a conformal Killing vector. Finally, we discuss these results for the existence of matter inheritance vector in the special cases of the above mentioned spacetimes.
\end{abstract}

Keyword: Timelike and Spacelike Matter Inheritance Vectors, EnergyMomentum Tensor

\section{Introduction}

General Relativity (GR) is the field theory of gravity which is highly nonlinear. The non-linearity of GR makes the field equations non-linear. Sym-

*e-mail: msharif@math.pu.edu.pk 
metries yield physical restrictions to the gravitational field which give not only the simplicity but also provide special physical effects in the field. GR provides a rich arena to use symmetries in order to understand the natural relation between geometry and matter furnished by Einstein field equations (EFEs).

Symmetry inheritance of a kinematical or dynamical quantity is defined [1] mathematically as

$$
£_{\xi} A=2 \alpha A,
$$

where $£_{\xi}$ is Lie derivative along $\xi, \xi\left(x^{a}\right)$ is the inheritance vector, $\alpha\left(x^{a}\right)$ is a scalar function, and $A$ is any of the following: $g_{a b}$ (metric tensor), $R_{b c d}^{a}$ (Riemann tensor), $R_{a b}$ (Ricci tensor), $T_{a b}$ (matter tensor) or geometric objects constructed by them. One can find all well-known inheritance symmetries by requiring the particular forms of the quantity $A$. For example, if we take $A=g_{a b}$, the above equation defines an inheritance symmetry defined by Conformal Killing Vector (CKV). If $A=R_{b c d}^{a}$, this defines Riemann inheritance symmetry or Curvature inheritance symmetry. If $A=R_{a b}$, this is Ricci inheritance symmetry and for $A=T_{a b}$, the equation defines the matter inheritance symmetry. In the case of inheritance symmetry of CKVs, the function $\alpha\left(x^{a}\right)$ is called the conformal factor and in the case of other inheritance symmetries, it is called the inheritance factor. When $\alpha=0$, all the inheritance cases reduce to the cases of collineations.

The matter inheritance symmetry (MIV) is defined by

$$
£_{\xi} T_{a b}=2 \alpha T_{a b},
$$

which can be expressed in component form as

$$
T_{a b ; c} \xi^{c}+T_{a c} \xi_{; b}^{c}+T_{b c} \xi_{; a}^{c}=2 \alpha T_{a b}
$$

The study of MIV is important as it helps in studying the invariance properties of a given geometrical object namely the Einstein tensor. This tensor plays an important role in the theory of GR, since it is related via EFEs to the material content of spacetime (represented by the matter tensor). Also, the symmetries of energy-momentum tensor provide conservation laws on matter field. They also help us to find out how the physical fields occurring in a certain region of spacetime reflect the symmetries of the metric.

A recent literature on inheritance symmetries in different spacetimes has attracted many people. Herrera and Ponce [2] have discussed CKVs in perfect and anisotropic fluids. Maartens et al. [3] have made a study of special 
conformal Killing vectors (SCKVs) in anisotropic fluids. Coley and Tupper [4] have considered spacetimes admitting SCKV and symmetry inheritance. Carot et al. [5] have discussed spacetimes with CKVs. Duggal [1,6] has studied curvature inheritance symmetry and timelike Ricci inheritance symmetry in fluid spacetimes.

Greenberg [7] was the first to introduce the theory of spacelike congruences in GR. It was further developed with applications by Mason and Tsamparlis [8], who considered spacelike CKVs in spacelike congruences. Yavuz and Yilmaz [9] considered inheriting CKVs and SCKVs in string cosmology (string cloud and string fluid). They also discussed solutions of string cosmology in static spherical symmetric spacetime via CKVs. Yilmaz et al. [10] and Baysal et al. [11] worked on the curvature inheritance symmetry and conformal collineations respectively in string cosmology. In his paper, Yilmaz [12] has considered timelike and spacelike Ricci collineations in string cloud. Baysal and Yilmaz [13] extended this work to spacelike Ricci inheritance vectors in string cosmology. In another paper [14], the same authors studied timelike and spacelike Ricci collineations in the model of string fluid.

Zeldovich [15] argued that the study of string cloud and string fluid models could give rise to density perturbations leading to the formation of galaxies. Kibble [16] shows the consistency in the existence of a large scale network of strings in the early universe and the today's observations of the universe. According to them the grand unification theories can also explain the presence of strings. Thus, it would be worth interesting to investigate the symmetry features of strings. The energy-momentum tensor associated with a perfect fluid has been widely studied in GR as a source of gravitational field, mainly to describe models of stars, galaxies and universes [17]. Also, it has been used to solve the EFEs using different prescriptions. Here the symmetry feature of the perfect fluid would be used.

In this paper, we extend the work for timelike MIVs and spacelike MIVs (SpMIVs) using string cosmology and perfect fluid. We shall also discuss the conditions for a MIV to be a CKV. The paper has been organised as follows. In section 2, we shall review some general results of timelike MIVs as well as SpMIVs to be used in the next sections. In sections 3 and 4, we shall solve the MIV equations and find out the necessary and sufficient conditions for timelike MIVs and SpMIVs in different fluid spacetimes to admit them. Also, we shall give the necessary and sufficient conditions for both timelike MIV and SpMIV when it is a CKV. In the last section, we shall conclude and discuss the results obtained. 


\section{Some General Results}

This section is devoted to discuss some general results about timelike MIVs as well as SpMIVs which will be used in later sections. Before discussing these results, we give the specific forms of the matter tensor to be used in this paper.

The energy-momentum tensor for a string cloud can be written as [18]

$$
T_{a b}=\rho u_{a} u_{b}-\lambda x_{a} x_{b}
$$

where $\rho$ is the rest energy for the cloud of strings with particles attached to them and $\lambda$ is the string tensor density and are related by $\rho=\rho_{p}+\lambda$. Here $\rho_{p}$ is particle energy density. This energy-momentum tensor represents a model of massive strings. Each massive string is formed by a geometric string with particles attached along its extension. This is the simplest model where we have particle and strings together.

The energy-momentum tensor for a string fluid [20] is

$$
T_{a b}=\rho_{s}\left(u_{a} u_{b}-x_{a} x_{b}\right)+q H_{a b},
$$

where $\rho_{s}$ is the string density, $q$ is the string tension and also pressure. $H_{a b}$ is screen projection operator and will be explained later. The energymomentum tensor for a perfect fluid [17] is

$$
T_{a b}=(\rho+p) u_{a} u_{b}+p g_{a b},
$$

where $\rho$ is the energy density and $\mathrm{p}$ is the pressure of fluid.

\subsection{Timelike Matter Inheritance Vectors}

Let $\xi^{a}=\xi u^{a}$, where $u^{a}$ is a unit timelike four-velocity vector orthogonal to the four-vector $x^{a}$ satisfying the following properties:

$$
x^{a} x_{a}=1, \quad u^{a} u_{a}=-1 \quad u^{a} x_{a}=0 .
$$

The vector $u_{a ; b}$ can be decomposed into its antisymmetric part, symmetric trace free part and trace as follows [20]

$$
u_{a ; b}=\omega_{a b}+\sigma_{a b}+\frac{\theta}{3} h_{a b}-\dot{u}_{a} u_{b}
$$


where $\dot{u}_{a}$ is the acceleration, $\omega_{a b}$ is rotation (rotational velocity), $\sigma_{a b}$ is shear (shear velocity) and $\theta$ is the expansion (expansion velocity). These quantities are mathematically defined as

$$
\begin{aligned}
\dot{u}_{a} & =u_{a ; n} u^{n}=\frac{D u_{a}}{D \tau}, \\
\omega_{a b} & =u_{[a ; b]}+\dot{u}_{[a} u_{b]}, \\
\sigma_{a b} & =u_{(a ; b)}+\dot{u}_{(a} u_{b)}-\frac{\theta}{3} h_{a b}, \\
\theta & =u_{; a}^{a} .
\end{aligned}
$$

The projection tensor, $h_{a b}=g_{a b}+u_{a} u_{b}$, has the following properties:

$$
h^{a b} x_{a}=x^{b}, \quad h^{a b} u_{a}=0, \quad h_{c}^{a} h_{b}^{c}=h_{b}^{a}, \quad h_{a b}=h_{b a}, \quad h_{a}^{a}=3 .
$$

When $\xi^{a}=\xi u^{a}$, we can re-write the matter inheritance symmetry as

$$
\dot{T}_{a b}+u^{c} T_{c(a} \ln \xi_{, b)}+T_{c(a} u_{; b)}^{c}=2 \alpha \xi^{-1} T_{a b} .
$$

The primary effect of a timelike CKV $\xi^{a}=\xi u^{a}$ is a well-known equation given by $£_{\xi} g_{a b}=2 \psi g_{a b}$ which is equivalent to the following conditions [21] (Proof is given in Appendix A)

$$
\begin{aligned}
\dot{u}_{a}-(\ln \xi)_{, a} & =u_{a}(\ln \xi)^{\cdot}=\psi \xi^{-1} u_{a} \\
\theta & =3 \psi \xi^{-1} \\
\sigma_{c d} & =0
\end{aligned}
$$

\subsection{Spacelike Matter Inheritance Vectors}

Let $\xi^{a}=\xi x^{a}$, where $x^{a}$ is a unit spacelike vector orthogonal to unit fourvelocity vector $u^{a}$ satisfying the following relations:

$$
x^{a} x_{a}=1, \quad u^{a} u_{a}=-1 \quad u^{a} x_{a}=0 .
$$

The vector $x_{a ; b}$ can be decomposed with respect to $u^{a}$ and $x^{a}$ in the following way $[22]$

$$
x_{a ; b}=A_{a b}+x_{a}^{*} x_{b}-\dot{x_{a}} u_{b}+u_{a}\left[x^{t} u_{t ; b}+\left(x^{t} \dot{u}_{t}\right) u_{b}-\left(x^{t} u_{t}^{*}\right) x_{b}\right],
$$


where $s_{\ldots}^{* \ldots}=s_{\ldots ; a}^{\ldots} x^{a}$ and $A_{a b}=H_{a}^{c} H_{b}^{d} x_{c ; d}$. Here $H_{a b}$ is screen projection operator perpendicular to both $u_{a}$ and $x_{a}$, given by

$$
H_{a b}=g_{a b}+u_{a} u_{b}-x_{a} x_{b} .
$$

This has the following properties:

$$
H^{a b} x_{a}=H^{a b} u_{a}=0, \quad H_{c}^{a} H_{b}^{c}=H_{b}^{a}, \quad H_{a b}=H_{b a}, \quad H_{a}^{a}=2 .
$$

We decompose $A_{a b}$ into its irreducible parts

$$
A_{a b}=S_{a b}+W_{a b}+\frac{1}{2} \theta^{*} H_{a b},
$$

where $S_{a b}=S_{b a}, \quad S_{a}^{a}=0$ is the traceless part of $A_{a b}, \theta^{*}$ is the trace of $A_{a b}$ and $W_{a b}=-W_{b a}$ is the rotation of $A_{a b}$. We can have the following relations:

$$
S_{a b}=H_{a}^{c} H_{b}^{d} x_{(c ; d)}-\frac{1}{2} \theta^{*} H_{a b}, \quad W_{a b}=H_{a}^{c} H_{b}^{d} x_{[c ; d]}, \quad \theta^{*}=H^{a b} x_{a ; b} .
$$

The term in square parentheses, in Eq.(19), can be written as

$$
-N_{b}+2 \omega_{t b} x^{t}+H_{b}^{t} \dot{x}^{t}
$$

where $N_{a}=H_{a}^{b}\left(\dot{x_{b}}-u_{b}^{*}\right)$ is the Greenberg vector and $\omega_{t b}$ is the rotational velocity. Using this value, it follows that Eq.(19) takes the form

$$
x_{a ; b}=A_{a b}+x_{a}^{*} x_{b}-\dot{x_{a}} u_{b}+H_{b}^{c} \dot{x}_{c} u_{a}+\left(2 \omega_{t b} x^{t}-N^{b}\right) u_{a} .
$$

Also, we have

$$
x^{t} u_{t ; b}=2 x^{t} u_{[t ; b]}+u_{b}^{*}=-2 \omega_{b t} x^{t}-\left(x_{t} \dot{u}^{t}\right) u_{b}+u_{b}^{*} .
$$

When $\xi^{a}=\xi x^{a}$, the matter inheritance symmetry, given in Eq.(3), can be written as

$$
T_{a b}^{*}+x^{c} T_{c(a} \ln \xi_{, b)}+T_{c(a} x_{; b)}^{c}=2 \alpha \xi^{-1} T_{a b} .
$$

It is known that the primary effect of a $\mathrm{SpCKV} \xi^{a}=\xi x^{a}$ is a wellknown equation given by $£_{\xi} g_{a b}=2 \psi g_{a b}$, which is equivalent to the following conditions [8] (Proof is given in Appendix B)

$$
\begin{aligned}
S_{a b} & =0, \\
x_{a}^{*}+(\ln \xi)_{, a} & =\frac{1}{2} \theta^{*} x_{a}, \\
\dot{x}^{a} u_{a} & =\frac{-1}{2} \theta^{*}, \\
N_{a} & =-2 \omega_{a b} x^{b}, \\
\psi & =\frac{1}{2} \xi \theta^{*}=\xi^{*} .
\end{aligned}
$$




\section{Timelike Matter Inheritance Vectors}

In this section, we shall prove the necessary and sufficient conditions for the existence of timelike MIV in the model of string cosmology and perfect fluid. In addition, we shall give the conditions for the existence of timelike MIV which is also a timelike CKV.

\subsection{String Cloud}

Theorem: The string cloud spacetime with energy-momentum tensor, given by Eq.(4), admits a timelike MIV $\xi^{a}=\xi u^{a}$ if and only if

$$
\begin{aligned}
2 \rho\left[\alpha \xi^{-1}-(\ln \xi)^{\cdot}\right] & =\dot{\rho}, \\
\dot{u}_{c}-(\ln \xi)_{, c}-(\ln \xi)^{\cdot} u_{c} & =0, \\
2 \lambda\left[\alpha \xi^{-1}-x_{t} u^{* t}\right] & =\dot{\lambda}, \\
2 \alpha \xi^{-1} \gamma_{a b}-2 \sigma_{t(a} \gamma_{b)}^{t}-2 \omega_{t(a} \gamma_{b)}^{t} & \\
-\frac{2}{3}\left[\theta \gamma_{a b}-h_{a b} \gamma^{c d} \sigma_{c d}-\lambda \sigma_{a b}\right] & =h_{a}^{c} h_{b}^{d} \dot{\gamma}_{c d},
\end{aligned}
$$

where

$$
\gamma^{a b}=\lambda\left[\frac{1}{3} h^{a b}-x^{a} x^{b}\right]
$$

Proof: First we assume that a timelike MIV exists in this spacetime and prove that the conditions given by Eq.(31)-(34) are satisfied.

When we substitute value of the energy-momentum tensor for string cloud from Eq.(4) in Eq.(14) and make use of Eq.(35), we have

$$
\begin{aligned}
\dot{\rho} u_{a} u_{b}-\frac{\dot{\lambda}}{3} h_{a b}+2 \rho\left[\dot{u}_{(a} u_{b)}-u_{(a}(\ln \xi)_{, b)}\right] & -\frac{2 \lambda}{3}\left[u_{(a ; b)}+\dot{u}_{(a} u_{b)}\right]+\dot{\gamma}_{a b}+2 \gamma_{t(a} u_{; b)}^{t} \\
& =2 \xi^{-1} \alpha\left[\rho u_{a} u_{b}-\frac{\lambda}{3} h_{a b}+\gamma_{a b}\right] .
\end{aligned}
$$

Contracting Eq.(36) in turn with $u^{a} u^{b}, u^{a} h_{c}^{b}, h^{a b}$ and $h_{c}^{a} h_{d}^{b}-\frac{1}{3} h^{a b} h_{c d}$, after some algebra, we obtain

$$
\begin{gathered}
\dot{\rho}+2 \rho\left[(\ln \xi)-\alpha \xi^{-1}\right]=0 \\
h_{c}^{b}\left[\dot{u}_{b}-(\ln \xi)_{, b}\right]=0 \\
\dot{\lambda}+2 \lambda\left[x_{c} u^{* c}-\alpha \xi^{-1}\right]=0 \\
h_{c}^{a} h_{d}^{b} \dot{\gamma}_{a b}=2 \alpha \xi^{-1} \gamma_{c d}-2 \sigma_{t(c} \gamma_{d)}^{t}-2 \omega_{t(c} \gamma_{d)}^{t}-\frac{2}{3}\left[\theta \gamma_{c d}-h_{c d} \gamma^{a b} \sigma_{a b}-\lambda \sigma_{c d}\right] .
\end{gathered}
$$


(i) Condition (31) is given by Eq.(37).

(ii) Expanding $h_{c}^{b}$ in Eq.(38) and multiplying by the rest of the expression, condition (32) turns out.

(iii) Condition (33) is the same as Eq.(39).

(iv) Eq.(40) gives condition (34).

Now we shall show that if the conditions (31)-(34) are satisfied, then there must exist a timelike MIV in a string cloud spacetime. In other words, we verify Eq.(36). When we substitute the values from Eqs.(31)-(33) in the left hand side of Eq.(36), we obtain

$$
2 \alpha \xi^{-1}\left[\rho u_{a} u_{b}-\frac{\lambda}{3} h_{a b}\right]+\frac{2 \lambda}{3} h_{a b} x_{t} u^{* t}-\frac{2 \lambda}{3}\left[\sigma_{a b}+\frac{\theta}{3} h_{a b}\right]+\dot{\gamma}_{a b}+2 \gamma_{t(a} u_{; b)}^{t}
$$

If we make use of Eq.(34) in the above expression together the value of $u_{t ; a}$ from Eq.(8), we finally have the right hand side of Eq.(36). Hence the conditions (31)-(34) are necessary and sufficient for a string cloud spacetime to admit a timelike MIV.

The necessary and sufficient conditions for the string cloud spacetime having a timelike MIV $\xi^{a}=\xi u^{a}$ which is also a timelike CKV are the same as given by Eqs.(15)-(17).

\subsection{String Fluid}

Theorem: The string fluid spacetime with energy-momentum tensor, given by Eq.(5), admits a timelike MIV $\xi^{a}=\xi u^{a}$ if and only if

$$
\begin{gathered}
\left(\rho_{s} u^{a} \xi\right)_{; a}=-\alpha T \\
\rho_{s}\left[\dot{u}_{a}-(\ln \xi)_{, a}-\theta u_{a}\right]=2 \alpha \xi^{-1} q u_{a}, \\
2 \alpha \xi^{-1} \gamma_{a b}-2 \sigma_{t(a} \gamma_{b)}^{t}-2 \omega_{t(a} \gamma_{b)}^{t} \\
-\frac{2}{3}\left[\theta \gamma_{a b}-h_{a b} \gamma^{c d} \sigma_{c d}+\left(2 q-\rho_{s}\right) \sigma_{a b}\right]=h_{a}^{c} h_{b}^{d} \dot{\gamma}_{c d},
\end{gathered}
$$

where

$$
\gamma^{a b}=\left(\rho_{s}+q\right)\left[\frac{1}{3} h^{a b}-x^{a} x^{b}\right] .
$$

Proof: First we suppose that a string fluid spacetime admits a timelike MIV and derive the above conditions. 
When we use Eqs.(5), (14) and (45), it follows that

$$
\begin{array}{r}
\dot{\rho}_{s} u_{a} u_{b}+\frac{1}{3}\left(2 \dot{q}-\dot{\rho}_{s}\right) h_{a b}+\frac{4}{3}\left(q+\rho_{s}\right) \dot{u}_{(a} u_{b)}+\dot{\gamma}_{a b} \\
-2 \rho_{s} u_{(a}(\ln \xi)_{, b)}+\frac{2}{3}\left(2 q-\rho_{s}\right) u_{(a ; b)}+2 \gamma_{c(a} u_{; b)}^{c} \\
=2 \alpha \xi^{-1}\left[\rho_{s} u_{a} u_{b}+\frac{h_{a b}}{3}\left(2 q-\rho_{s}\right)+\gamma_{a b}\right] .
\end{array}
$$

If we contract Eq.(46) with $u^{a} u^{b}, u^{a} h_{c}^{b}, h^{a b}$ and $h_{c}^{a} h_{d}^{b}-\frac{1}{3} h^{a b} h_{c d}$, after some tedious computation, we obtain

$$
\begin{aligned}
\dot{\rho}_{s}+2 \rho_{s}\left[(\ln \xi)-\alpha \xi^{-1}\right] & =0 \\
h_{c}^{b}\left[\dot{u}_{b}-(\ln \xi)_{, b}\right] & =0 \\
\left(2 \dot{q}-\dot{\rho}_{s}\right)+2 \gamma^{a b} \sigma_{a b}+\frac{2}{3}\left(2 q-\rho_{s}\right) \theta & =2 \alpha \xi^{-1}\left[2 q-\rho_{s}\right] \\
2 \alpha \xi^{-1} \gamma_{a b}-2 \sigma_{t(a} \gamma_{b)}^{t}-2 \omega_{t(a} \gamma_{b)}^{t} & \\
-\frac{2}{3}\left[\theta \gamma_{a b}-h_{a b} \gamma^{c d} \sigma_{c d}+\left(2 q-\rho_{s}\right) \sigma_{a b}\right] & =h_{a}^{c} h_{b}^{d} \dot{\gamma}_{c d} .
\end{aligned}
$$

(i) Using the energy-momentum conservation law, $T_{; b}^{a b}=0$, in the case of string fluid, we obtain $\dot{q}=\dot{\rho}_{s}$ and $\dot{\rho}_{s}=-\frac{2}{3}\left(\rho_{s}+q\right) \theta-\gamma^{a b} \sigma_{a b}$ which implies that

$$
\dot{q}=-\frac{2}{3}\left(\rho_{s}+q\right) \theta-\gamma^{a b} \sigma_{a b}
$$

Replacing this value of $\dot{q}$ in Eq.(49), we have

$$
\dot{\rho}_{s}=-2 \rho_{s} \theta-2 \alpha \xi^{-1}\left(2 q-\rho_{s}\right)
$$

Comparing Eqs.(47) and (52), we have

$$
\rho_{s}(\ln \xi)^{\cdot}=\rho_{s} \theta+2 \alpha \xi^{-1} q
$$

which finally gives

$$
\left(\rho_{s} u^{a} \xi\right)_{; a}=-\alpha T
$$

This yields the first condition given by Eq.(42).

(ii) Expanding Eq.(48), we have

$$
\dot{u}_{c}-(\ln \xi) \cdot u_{c}-(\ln \xi)_{, c}=0 .
$$

Substituting the value of $\rho_{s}(\ln \xi)$. from Eq.(53) in the above equation, we obtain the condition (43). 
(iii) Condition (44) is the same as Eq.(50).

To prove that the conditions (42)-(44) are sufficient, we shall take the left hand side of Eq.(46) given by

$$
\begin{gathered}
\dot{\rho}_{s} u_{a} u_{b}+\frac{1}{3}\left(2 \dot{q}-\dot{\rho}_{s}\right) h_{a b}+\frac{4}{3}\left(q+\rho_{s}\right) \dot{u}_{(a} u_{b)}+\dot{\gamma}_{a b} \\
-2 \rho_{s} u_{(a}(\ln \xi)_{, b)}+\frac{2}{3}\left(2 q-\rho_{s}\right) u_{(a ; b)}+2 \gamma_{c(a} u_{; b)}^{c} .
\end{gathered}
$$

Using Eq.(45) and the conditions (43)-(44), it follows that

$$
\begin{array}{r}
{\left[\dot{\rho}_{s}+2 \rho_{s} \theta\right] u^{a} u^{b}+\frac{1}{3}\left(2 \dot{q}-\dot{\rho}_{s}\right) h_{a b}+4 \alpha \xi^{-1} q u_{a} u_{b}+} \\
2 \alpha \xi^{-1} \gamma_{a b}-\frac{2}{3}\left[\left(2 \rho_{s}-q\right) \sigma_{a b}-h_{a b} \gamma^{c d} \sigma_{c d}\right]
\end{array}
$$

Also, Eqs.(42) and (43) imply that

$$
\dot{\rho}_{s}+2 \rho_{s} \theta=2 \alpha \xi^{-1}\left(\rho_{s}-2 q\right) .
$$

Thus the expression (55) becomes

$$
2 \alpha \xi^{-1}\left[\rho_{s} u_{a} u_{b}+\gamma_{a b}\right]+\frac{1}{3} h_{a b}\left[\left(2 \dot{\rho}_{s}-\dot{q}\right)+\frac{2}{3}\left(2 \rho_{s}-q\right) \theta+2 \gamma^{c d} \sigma_{c d}\right] .
$$

Now by using Eq.(51), it takes the form

$$
2 \xi^{-1} \alpha\left[\rho_{s} u_{a} u_{b}+\frac{h_{a b}}{3}\left(2 q-\rho_{s}\right)+\gamma_{a b}\right]
$$

which is the right hand side of the Eq.(46). Hence the conditions (42)-(44) are sufficient as well.

The string fluid spacetime admits a timelike MIV $\xi^{a}=\xi u^{a}$ which is also a timelike CKV if and only if

$$
\begin{aligned}
-\frac{\rho_{s} \psi}{q} & =\alpha \\
\sigma_{c d} & =0 \\
\theta & =3 \psi \xi^{-1}
\end{aligned}
$$

The proof is straightforward and it follows directly by the comparison of Eqs.(15)-(17) and Eqs.(42)-(44). 


\subsection{Perfect Fluid}

Theorem: The perfect fluid spacetime with energy-momentum tensor, given by Eq.(6), admits a timelike MIV $\xi^{a}=\xi u^{a}$ if and only if

$$
\begin{aligned}
\rho & =2 \rho\left[\alpha \xi^{-1}-(\ln \xi)^{\cdot}\right] \\
\dot{u}_{c}-(\ln \xi)_{, c}-u_{c}(\ln \xi)^{\cdot} & =0 \\
\dot{p} & =2 p\left(\alpha \xi^{-1}-\frac{1}{3} \theta\right), \\
p \sigma_{a b} & =0 .
\end{aligned}
$$

Proof: We first assume that timelike MIV exist and prove the above conditions. From Eqs.(6) and (14), we get

$$
\begin{array}{r}
(\rho+p) \cdot u_{a} u_{b}+\dot{p} g_{a b}+2(\rho+p) \dot{u}_{(a} u_{b)}-2 \rho u_{(a}(\ln \xi)_{, b)}-2 p u_{(a ; b)} \\
=2 \alpha \xi^{-1}\left[(\rho+p) u_{a} u_{b}+p g_{a b}\right] .
\end{array}
$$

Contracting Eq.(63) in turn with $u^{a} u^{b}, u^{a} h_{c}^{b}, h^{a b}$ and $h_{c}^{a} h_{d}^{b}-\frac{1}{3} h^{a b} h^{c d}$, we have

$$
\begin{aligned}
\rho & =2 \rho\left[\alpha \xi^{-1}-(\ln \xi)^{\cdot}\right] \\
\dot{u}_{c}-(\ln \xi)_{, c}-(\ln \xi) \cdot u_{c} & =0, \\
3 \dot{p}+2 \theta p & =6 \alpha \xi^{-1} p, \\
p\left[\left(h_{c}^{a} h_{d}^{b}-\frac{1}{3} h^{a b} h^{c d}\right)\left(u_{a ; b}+u_{b ; a}\right)\right] & =0 .
\end{aligned}
$$

(i) Condition (59) is followed by Eq.(64)

(ii) Eq.(65) is the same as condition (60).

(iii) Condition (61) follows by Eq.(66).

(iv) Substituting the values of $u_{a ; b}$ and $h_{b}^{a}$ in Eq.(67), we have the condition given by Eq.(62).

Now we shall prove that if the conditions (59)-(62) are satisfied, then the perfect fluid spacetime admits a MIV. Consider the left hand side of Eq.(63) given by

$$
(\rho+p) \cdot u_{a} u_{b}+\dot{p} g_{a b}+2(\rho+p) \dot{u}_{(a} u_{b)}-2 \rho u_{(a}(\ln \xi)_{, b)}+2 p u_{(a ; b)} .
$$


Substituting the value of $u_{a ; b}$ and making use of Eqs.(59)-(62), we have

$$
2 \alpha \xi^{-1}\left[\rho u_{a} u_{b}+p h_{a b}\right] .
$$

Hence the conditions are necessary as well as sufficient for the perfect fluid to admit a timelike MIV.

The perfect fluid spacetime admits a timelike MIV $\xi^{a}=\xi u^{a}$ which is also a timelike CKV if and only if the conditions given by Eqs.(15)-(17) are satisfied.

\section{Spacelike Matter Inheritance Vectors}

This section deals with the necessary and sufficient conditions when it admits SpMIV for string cosmology and perfect fluid.

\subsection{String Cloud}

Theorem: The string cloud spacetime with energy-momentum tensor, admits a MIV $\xi^{a}=\xi x^{a}$ if and only if

$$
\begin{aligned}
\omega_{a b} x^{b} & =0 \\
\lambda\left[x_{a}^{*}+(\ln \xi)_{, a}\right] & =\lambda x_{a}(\ln \xi)^{*}, \\
\rho^{*} & =-2 \rho\left[x_{a} \dot{u}^{a}-\alpha \xi^{-1}\right], \\
\lambda^{*} & =-2 \lambda\left[(\ln \xi)^{*}-\alpha \xi^{-1}\right] .
\end{aligned}
$$

Proof: It follows from Eqs.(4) and (25) that

$$
\begin{array}{r}
\rho^{*} u_{a} u_{b}-\lambda^{*} x_{a} x_{b}+2 \rho_{s} u_{(a} u_{b)}^{*}-2 \lambda x_{(a} x_{b)}^{*}-2 \lambda x_{(a}(\ln \xi)_{, b)}-2 \rho x_{c} u_{(a} u_{; b)}^{c} \\
=2 \xi^{-1} \alpha\left[\rho u_{a} u_{b}-\lambda x_{a} x_{b}\right] .
\end{array}
$$

When we make contraction of Eq.(72) with $u^{a} u^{b}, u^{a} x^{b}, u^{a} H_{c}^{b}, x^{a} x^{b}$ and $x^{a} H_{c}^{b}$ in turn, the following equations turn out

$$
\begin{aligned}
\rho^{*}+2 \rho\left[x_{a} \dot{u}^{a}-\alpha \xi^{-1}\right] & =0, \\
\lambda\left[(\ln \xi)^{\cdot}-x_{a} u^{* a}\right] & =0, \\
H_{a}^{b}\left[u_{b}^{*}-x_{c} u_{; b}^{c}\right] & =0, \\
\lambda^{*}+2 \lambda\left[(\ln \xi)^{*}-\alpha \xi^{-1}\right] & =0, \\
\lambda H_{a}^{b}\left[x_{b}^{*}+(\ln \xi)_{, b}\right] & =0 .
\end{aligned}
$$


Now we check the consistency of the necessary and sufficient conditions given by Eqs.(68)-(71).

(i) Substituting the value of $x_{c} u_{; b}^{c}$ from Eq.(24) in Eq.(75), we obtain the condition given by Eq.(68).

(ii) Firstly, we expand Eq.(77) and then making use of Eq.(74), we get the condition (69).

(iii) Obviously, Eq.(73) implies the condition given by Eq.(70) directly.

(iv) Similarly, Eq.(76) follows the condition (71) directly.

Notice that the conditions given by Eqs.(68)-(71) satisfy Eq.(72). Hence the conditions (68)-(71) are the necessary and sufficient conditions for a vector $\xi^{a}=\xi x^{a}$ to be SpMIV.

It is mentioned here that a SpMIV $\xi^{a}=\xi x^{a}$ in a string cloud spacetime is also a SpCKV if and if

$$
\begin{aligned}
N_{a} & =0, \\
S_{a b} & =0, \\
\lambda(\ln \xi)^{*} & =\frac{\lambda \theta^{*}}{2}, \\
x_{a} \dot{u}^{a} & =\frac{1}{2} \theta^{*}, \\
\psi & =\xi^{*}=\frac{1}{2} \xi \theta^{*} .
\end{aligned}
$$

These can be easily verified by comparing Eqs.(26)-(30) and Eqs.(68)-(71).

\subsection{String Fluid}

Theorem: The string fluid spacetime with energy-momentum tensor has a MIV $\xi^{a}=\xi x^{a}$ if and only if

$$
\begin{aligned}
\rho_{s} \omega_{a b} x^{b} & =\frac{1}{2} q N_{a}, \\
q S_{a b} & =0, \\
x_{a}^{*}+(\ln \xi)_{; a}-\left(x_{b} \dot{u}^{b}\right) x_{a} & =0, \\
\rho_{s} \theta^{*} & =-2 \alpha q \xi^{-1}, \\
{\left[\rho_{s} \xi x^{a}\right]_{; a} } & =-\alpha T .
\end{aligned}
$$


Proof: If we make use of Eqs.(5) and (25), we can write

$$
\begin{array}{r}
\left.\rho_{s}^{*}\left[u_{a} u_{b}-x_{a} x_{b}\right]+q^{*} H_{a b}+2 q x_{(a ; b)}-2 \rho_{s} x_{(a}(\ln \xi) ; b\right) \\
+2\left(\rho_{s}+q\right)\left[u_{(a} u_{b)}^{*}-x_{c} u_{(a} u_{; b}^{c}-x_{(a} x_{b)}^{*}\right] \\
=2 \xi^{-1} \alpha\left[\left(\rho_{s}+q\right)\left(u_{a} u_{b}-x_{a} x_{b}\right)+q g_{a b}\right] .
\end{array}
$$

Now we make contraction of Eq.(88) with $u^{a} u^{b}, u^{a} x^{b}, u^{a} H_{c}^{b}, x^{a} x^{b}, x^{a} H_{c}^{b}, H^{a b}$ and $H_{c}^{a} H_{d}^{b}-\frac{1}{2} H^{a b} H_{c d}$ in turn, the following equations are obtained

$$
\begin{aligned}
\rho_{s}^{*}+2 \rho_{s}\left[x_{a} \dot{u}^{a}-\alpha \xi^{-1}\right] & =0 \\
(\ln \xi)+x_{a}^{*} u^{a} & =0 \\
q H_{a}^{b} \dot{x}_{b}-\left(\rho_{s}+q\right) H_{a}^{b} u_{b}^{*}+\rho_{s} H_{a}^{b} x^{c} u_{c ; b} & =0 \\
\rho_{s}^{*}+2 \rho_{s}\left[(\ln \xi)^{*}-\alpha \xi^{-1}\right] & =0 \\
H_{a}^{b}\left[x_{b}^{*}+(\ln \xi)_{, b}\right] & =0 \\
q^{*}+q\left(\theta^{*}-2 \alpha \xi^{-1}\right) & =0 \\
q S_{a b} & =0 .
\end{aligned}
$$

Let us now satisfy the necessary and sufficient conditions given by Eqs.(83)(87).

(i) If we replace the value of $x^{c} u_{c ; b}$ in Eq.(91), we obtain Eq.(83).

(ii) It is obvious that Eq.(95) implies Eq.(84).

(iii) After expanding Eq.(93) and using Eq.(90), we arrive at

$$
x_{a}^{*}+(\ln \xi)_{, a}-(\ln \xi)^{*} x_{a}=0 .
$$

Now subtraction of Eq.(92) from Eq.(89) yields

$$
(\ln \xi)^{*}=x_{a} \dot{u}^{a} .
$$

When we use Eq.(97) in Eq.(96), we get the condition given by Eq.(85).

(iv) The energy-momentum conservation equation for string fluid gives the following result

$$
q^{*}=-\left(\rho_{s}+q\right) \theta^{*}
$$

When we replace this value in Eq.(94), we get Eq.(86). 
(v) Since $\theta^{*}=H^{a b} x_{a ; b}$ and hence $x_{a} \dot{u}^{a}=x_{; a}^{a}-\theta^{*}$. When we substitute this value in Eq.(97), we get

$$
(\ln \xi)^{*}=\left(x_{; a}^{a}-\theta^{*}\right) .
$$

If one of the terms $\rho_{s}(\ln \xi)^{*}$ in Eq.(92) is replaced by Eq.(98), and condition (86) is used, then Eq.(92) may be written as

$$
\left(\rho_{s}\right)_{; a} \xi x^{a}+\rho_{s}\left(\xi_{; a} x^{a}+\xi x_{; a}^{a}\right)=2 \alpha\left(\rho_{s}-q\right) .
$$

Substituting $2\left(q-\rho_{s}\right)=T$ in the above equation, we obtain the condition given by Eq.(87).

Now we explore the conditions for the string fluid when a MIV is also a SpCKV. The string fluid spacetime admits a SpMIV $\xi^{a}=\xi x^{a}$, which is also a SpCKV if and only if

$$
\begin{aligned}
\left(\rho_{s}+q\right) N_{a} & =0 \\
S_{a b} & =0 \\
\xi^{*} & =\psi=\frac{1}{2} \xi \theta^{*}, \\
\alpha & =-\frac{\psi \rho_{s}}{q} \\
x_{a} \dot{u}^{a} & =\frac{1}{2} \theta^{*} .
\end{aligned}
$$

The proof of these results can be performed by the comparison of Eqs.(26)(30) and Eqs.(83)-(87). It is to be noted that in Eq.(102), $\alpha$ turns out to be the same as in Eq.(56).

\subsection{Perfect Fluid}

Theorem: The perfect fluid spacetime with energy-momentum tensor posesses a MIV $\xi^{a}=\xi x^{a}$ if and only if

$$
\begin{aligned}
p S_{a b} & =0, \\
\rho \omega_{a c} x^{c} & =\frac{1}{2} p N_{a}, \\
p\left[x_{a}^{*}+(\ln \xi)_{, a}-(\ln \xi)^{*} x_{a}\right] & =0, \\
2 p(\ln \xi) * & =p \theta^{*}, \\
\rho^{*} & =2 \rho\left[\alpha \xi^{-1}-x_{c} \dot{u}^{c}\right], \\
p^{*} & =p\left(2 \alpha \xi^{-1}-\theta^{*}\right) .
\end{aligned}
$$


Proof: First we assume that a perfect fluid spacetime admits a SpMIV and show that the conditions (104)-(109) can be obtained.

From Eqs.(6) and (25), we get

$$
\begin{array}{r}
(\rho+p)^{*} u_{a} u_{b}+p^{*} g_{a b}+2 p\left[x_{(a}(\ln \xi)_{, b)}+x_{(a ; b)}\right] \\
+2(\rho+p)\left[u_{(a} u_{b)}^{*}-x_{t} u_{(a} u_{; b)}^{t}\right]=2 \alpha \xi^{-1}\left[(\rho+p) u_{a} u_{b}+p g_{a b}\right] .
\end{array}
$$

Contracting Eq.(110) in turn with $u^{a} u^{b}, u^{a} x^{b}, u^{a} H_{c}^{b}, x^{a} x^{b}, x^{a} H_{c}^{b}, H^{a b}$ and $H_{c}^{a} H_{d}^{b}-\frac{1}{2} H^{a b} H_{c d}$, we have

$$
\begin{aligned}
\rho^{*}+2 \rho\left[x_{t} \dot{u}^{t}-\alpha \xi^{-1}\right] & =0, \\
p\left[(\ln \xi)+x_{a}^{*} u^{a}\right] & =0, \\
(\rho+p) H_{a}^{b} u_{b}^{*}-\rho H_{a}^{b} x^{t} u_{t ; b}-p H_{a}^{b} \dot{x_{b}} & =0, \\
p *+2 p\left[(\ln \xi)^{*}-\alpha \xi^{-1}\right] & =0, \\
p H_{a}^{b}\left[x_{b}^{*}+(\ln \xi)_{, b}\right] & =0, \\
p^{*}+p\left(\theta^{*}-2 \alpha \xi^{-1}\right) & =0, \\
p S_{a b} & =0 .
\end{aligned}
$$

(i) Condition (104) is given by Eq.(117).

(ii) Condition (105) is derived from Eq.(113) by substituting the value of $x^{t} u_{t ; b}$ from Eq. $(24)$.

(iii) By expanding Eq.(115) and using Eq.(112) we get condition given by Eq.(106).

(iv) Subtracting Eq.(114) from Eq.(116) gives condition (107).

$(v)$ Conditions (108) and (109) are derived directly from Eqs.(111) and (116) respectively.

Conversely, if the above conditions are satisfied then we show that in the perfect fluid spacetime SpMIV exists.

Consider the left hand side of Eq.(110),

$$
(\rho+p)^{*} u_{a} u_{b}+p^{*} g_{a b}+2 p\left[x_{(a}(\ln \xi)_{, b)}+x_{(a ; b)}\right]+2(\rho+p)\left[u_{(a} u_{b)}^{*}-x_{t} u_{(a} u_{; b)}^{t}\right] .
$$


If we use the expansion of $x_{a ; b}$ from Eq.(23), and also use the conditions (104), (108) and (109), we have

$$
\begin{array}{r}
2 \alpha \xi^{-1}\left[(\rho+p) u_{a} u_{b}+p g_{a b}\right]-2 \rho u_{a} u_{b}\left(x_{t} \dot{u}^{t}\right)-p \theta^{*} h_{a b} \\
+2(\rho+p)\left[2 u_{(a} w_{b) t} x^{t}+\left(x_{t} \dot{u}^{t}\right) u_{a} u_{b}\right]+p\left[\theta^{*} H_{a b}-2\left(x_{t} \dot{u}^{t}\right) u_{a} u_{b}\right. \\
\left.-4 u_{(a} \omega_{b) t} x^{t}-2 u_{(a} N_{b)}+2 x_{(a} x_{b)}^{*}+2 x_{(a}(\ln \xi)_{, b)}\right] .
\end{array}
$$

Using Eqs.(105), (106) and (107), we obtain

$$
2 \alpha \xi^{-1}\left[(\rho+p) u_{a} u_{b}+p g_{a b}\right]
$$

which implies the right hand side of Eq.(110). Hence the conditions are necessary as well as sufficient.

The perfect fluid spacetime admits a SpMIV $\xi^{a}=\xi x^{a}$ which is also a SpCKV if and only if the following conditions are satisfied.

$$
\begin{aligned}
S_{a b} & =0, \\
(\ln \xi)^{*} & =\frac{\theta^{*}}{2}, \\
2(\rho+p) \omega_{a c} x^{c} & =0, \\
x_{a} \dot{u}^{a} & =\frac{1}{2} \theta^{*}, \\
\psi & =\frac{1}{2} \xi \theta^{*}=\xi^{*} .
\end{aligned}
$$

The proof follows by the comparison of Eqs.(104)-(109) and Eqs.(26)-(30).

\section{Summary and Discussion}

This paper deals with the fundamental question of determining when the symmetries of the geometry is inherited by all the source terms of a prescribed matter tensor of EFEs. Physically, there is a close connection of inheriting CKVs with the relativistic thermodynamics of fluids since for a distribution of massless particles in equilibrium the inverse temperature function is inheriting $\mathrm{CKV}$.

In this paper, we have found the necessary and sufficient conditions for the existence of timelike MIVs and SPMIVs in string cosmology and perfect

fluid spacetime. In the case of timelike MIVs, we obtain 4 conditions for the 
string cloud model, 3 conditions for the string fluid model and 4 conditions for the perfect fluid model which are necessary as well as sufficient for the existence of such vectors. In the case of SpMIVs, we obtain 5 conditions for the string cloud, 4 for the string fluid and 6 for perfect fluid spacetime. We have also compared these conditions with the conditions of CKV to get the conditions of MIVs which is also a CKV. In the following we discuss these conditions in detail for the specific cases.

\subsection{Timelike MIVs in String Cloud}

1. When $\lambda=0$, or $\rho=\rho_{p}$, the case reduces to the case of cloud of particles and the conditions are

$$
\begin{aligned}
2 \rho_{p}\left[\alpha \xi^{-1}-(\ln \xi) \cdot\right] & =\dot{\rho}_{p} \\
\dot{u}_{c}-(\ln \xi)_{, c}-(\ln \xi) \cdot u_{c} & =0 .
\end{aligned}
$$

2. When $\rho_{p}=0$ or $\rho=\lambda$, we get the conditions for the existence of timelike MIV in geometric strings. These are

$$
\begin{aligned}
2 \rho\left[\alpha \xi^{-1}-(\ln \xi)^{\cdot}\right] & =\dot{\rho}, \\
\dot{u}_{a}-(\ln \xi)_{, a}-(\ln \xi)^{\cdot} u_{a} & =0, \\
x_{t} u^{* t} & =(\ln \xi)^{\prime}, \\
2 \alpha \xi^{-1} \gamma_{a b}-2 \sigma_{t(a} \gamma_{b)}^{t}-2 \omega_{t(a} \gamma_{b)}^{t} & \\
-\frac{2}{3}\left[\theta \gamma_{a b}-h_{a b} \gamma^{c d} \sigma_{c d}-\lambda \sigma_{a b}\right] & =h_{a}^{c} h_{b}^{d} \dot{\gamma}_{c d} .
\end{aligned}
$$

\subsection{Timelike MIVs in String Fluid}

1. Eq.(42) is the kinematic equation for the string fluid.

2. When $q=0$, the case becomes the case of pure strings with the following necessary and sufficient conditions to admit a MIV

$$
\begin{gathered}
\left(\rho_{s} u^{a} \xi\right)_{; a}=2 \alpha \rho_{s} \\
\dot{u}_{a}-(\ln \xi)_{a}-\theta u_{a}=0 \\
2 \alpha \xi^{-1} \gamma_{a b}-2 \sigma_{t(a} \gamma_{b)}^{t}-2 \omega_{t(a} \gamma_{b)}^{t} \\
-\frac{2}{3}\left[\theta \gamma_{a b}-h_{a b} \gamma^{c d} \sigma_{c d}-\rho_{s} \sigma_{a b}\right]=h_{a}^{c} h_{b}^{d} \dot{\gamma}_{c d}
\end{gathered}
$$


The conditions (129) and (130) imply that $(\ln \xi)^{\cdot}=\theta=u^{* t} x_{t}$ and

$$
2 \rho_{s}\left[\alpha \xi^{-1}-(\ln \xi)^{\cdot}\right]=\dot{\rho}_{s} .
$$

This shows that the above conditions for string cloud and string fluid are the same for $\lambda=\rho$ and $\rho=\rho_{s}$.

\subsection{Timelike MIVs in Perfect fluid}

1. Eq.(63) gives either the shear velocity or the pressure vanishes. When $p=0$, it reduces to the case of dust and the conditions become similar to the case of string cloud for $\rho=\rho_{p}$. The vanshing of shear velocity implies that the stress is zero.

2. When $\rho=p$, it gives the stiff matter and the corresponding conditions are

$$
\begin{aligned}
\dot{p} & =2 p\left[\alpha \xi^{-1}-(\ln \xi)^{\cdot}\right], \\
\dot{u}_{c}-(\ln \xi)_{, c} & =u_{c}(\ln \xi)^{\cdot} \\
\dot{p} & =2 p\left(\alpha \xi^{-1}-\frac{1}{3} \theta\right), \\
\sigma_{a b} & =0 .
\end{aligned}
$$

We also obtain the same conditions as above for $\rho=3 p$, i.e., the radiation case and for $\rho=-p$, i.e., the vacuum case.

It is to be noted that in all the cases (string cosmology and perfect fluid), the conditions for a timelike MIV which is also a timelike CKV are the same as given in Eqs.(15)-(17).

\subsection{Spacelike MIVs in String Cloud}

1. Eq.(68) gives

$$
\omega_{a b} x^{b}=0 .
$$

Since $\omega_{a b}=\eta_{a b c d} \omega^{c} u^{d}$, we have by contracting Eq.(136) with $\eta^{a t e f} \omega_{e} u_{f}$ that

$$
x^{a}=\left[\left(\omega_{b} x^{b}\right) / \omega^{2}\right] \omega^{a} .
$$

Also, both $x^{a} \neq 0$ and $\omega^{a} \neq 0$, it follows that $x^{a}= \pm \omega^{a} / \omega$ and the curves are material curves. 
2. If the string tensor density is zero, i.e., $\lambda=0$, then $\rho=\rho_{p}+\lambda$ implies that $\rho=\rho_{p}$. The case reduces to the cloud of particles and the conditions of SpMIV given by Eqs.(68)-(71) turn out to be

$$
\begin{aligned}
\omega_{a c} x^{c} & =0, \\
\rho_{p}^{*} & =2 \rho_{p}\left[\alpha \xi^{-1}-x_{c} \dot{u}^{c}\right] .
\end{aligned}
$$

When we compare the above conditions with Eqs.(15)-(17), the conditions for a SpMIV which is also a SpCKV change into

$$
\begin{aligned}
S_{a b} & =0 \\
x_{c}^{*}+(\ln \xi)_{, c} & =\frac{1}{2} \theta^{*} x_{c}, \\
N_{a} & =0 \\
x_{a} u^{a} & =\frac{1}{2} \theta^{*}, \\
\psi & =\frac{1}{2} \xi=\xi^{*} .
\end{aligned}
$$

4. When $\rho_{p}=0$, i.e., particle energy density vanishes, then $\rho=\lambda$. This is the case of geometric strings or Nambu strings [18]. The conditions for the existence of SpMIV become

$$
\begin{aligned}
x_{c}^{*}+(\ln \xi)_{, c} & =x_{c}(\ln \xi)^{*}, \\
\omega_{a c} x^{c} & =0, \\
\rho^{*} & =2 \rho\left[\alpha \xi^{-1}-x_{c} \dot{u^{c}}\right], \\
x_{c} \dot{u}^{c} & =(\ln \xi)^{*} .
\end{aligned}
$$

Also, the conditions for a SpMIV which is also a SpCKV takes the form

$$
\begin{aligned}
S_{a b} & =0, \\
N_{a} & =0 \\
(\ln \xi)^{*} & =\frac{\theta^{*}}{2}=x_{c} \dot{u}^{c}, \\
\psi & =\frac{1}{2} \xi \theta^{*}=\xi^{*} .
\end{aligned}
$$




\subsection{Spacelike MIVs in String Fluid}

1. It follows from Eq.(84) that either $q=0$ or $S_{a b}=0$. If $q=0$, the case reduces to the case of pure strings and $S_{a b}=0$ implies that the shear of a spacelike congruence, generated by a MIV, vanishes.

2. If we take $\omega=0$, Eq.(83) implies that $q N_{a}=0$ which gives two possibilities either $q=0$ or $N_{a}=0$. The case $q=0$ is the same as above and $N_{a}=0$ implies the integral curve, i.e., $x^{a}$ are material curves and the string fluid forms the two-surface.

3. When we assume that $\omega \neq 0$ and $N_{a}=0$, we get $x^{a}= \pm \omega^{a} / \omega$ and curves are material curves.

\subsection{Spacelike MIVs in Perfect Fluid}

1. If $N_{a}=0$, i.e., the integral curves are material curves, then the condition (105) gives $\rho \omega_{a c} x^{c}=0$ which implies that either $\rho=0$ or $\omega_{a c} x^{c}=0$. If $\omega_{a c} x^{c}=0$, we obtain the similar results as for the case of string cloud.

2. When $p=0$, the case reduces to the case of dust. In this case we get the same necessary and sufficient conditions for the existence of the MIV as in the case of cloud of particles with $\rho_{p}=\rho$.

3. The case $\rho=p$ in a perfect fluid implies stiff matter. The necessary and sufficient conditions of the existence of MIV in this case are

$$
\begin{aligned}
S_{a b} & =0, \\
\omega_{a c} x^{c} & =\frac{1}{2} N_{a}, \\
x_{a}^{*}+(\ln \xi)_{, a}-(\ln \xi)^{*} x_{a} & =0, \\
2(\ln \xi) * & =\theta^{*}, \\
p^{*} & =2 p\left[\alpha \xi^{-1}-x_{c} \dot{u}^{c}\right], \\
p^{*} & =p\left(2 \alpha \xi^{-1}-\theta^{*}\right) .
\end{aligned}
$$

4. $\rho=3 p$ implies the case of radiation and the conditions of the existence of MIV reduce to

$$
S_{a b}=0
$$




$$
\begin{aligned}
\omega_{a c} x^{c} & =\frac{1}{6} N_{a}, \\
x_{a}^{*}+(\ln \xi)_{, a}-(\ln \xi)^{*} x_{a} & =0, \\
2(\ln \xi) * & =\theta^{*}, \\
p^{*} & =2 p\left[\alpha \xi^{-1}-x_{c} \dot{u}^{c}\right], \\
p^{*} & =p\left(2 \alpha \xi^{-1}-\theta^{*}\right) .
\end{aligned}
$$

5. $\rho=-p$ in a perfect fluid gives vacuum state. The necessary and sufficient conditions of the existence of MIV in this case are

$$
\begin{aligned}
S_{a b} & =0, \\
N_{a}+2 \omega_{a c} x^{c} & =0, \\
x_{a}^{*}+(\ln \xi)_{, a}-(\ln \xi)^{*} x_{a} & =0, \\
2(\ln \xi) * & =\theta^{*}, \\
p^{*} & =2 p\left[\alpha \xi^{-1}-x_{c} \dot{u}^{c}\right], \\
p^{*} & =p\left(2 \alpha \xi^{-1}-\theta^{*}\right) .
\end{aligned}
$$

The necessary and sufficient conditions for the existence of MIV which is also a CKV are the same in the cases (3), (4) and (5) given as

$$
\begin{aligned}
S_{a b} & =0, \\
(\ln \xi)^{*} & =\frac{\theta^{*}}{2}, \\
\omega_{a c} x^{c} & =0, \\
x_{a} u^{a} & =\frac{1}{2} \theta^{*}, \\
\psi & =\frac{1}{2} \xi \theta^{*}=\xi^{*} .
\end{aligned}
$$

It is mentioned here that for $\alpha=0$, we obtain the conditions of matter collineations in each case of spacelike and timelike MIVs for the models of string cloud, string fluid and perfect fluid.

We have obtained the conditions for the existence of MIVs in the models of string cloud, string fluid and perfect fluid. These conditions can be used as restriction for the EFEs. Since the non-linearity of EFEs ceases to extract their exact solution, the restricted equations may give interesting solution in respective spacetimes. Matter inheritance symmetry for null fluid spacetimes can be defined. Cylindrically symmetric and spherically symmetric 
spacetimes can be classified by the MIVs. It would be worth interesting to look for the necessary and sufficient conditions for the existence of null MIVs in different cosmological models.

\section{Appendix A}

\section{Conditions for Timelike CKVs}

Here we prove the necessary and sufficient conditions for a timelike CKV.

Theorem: The primary effect of a timelike CKV $\xi^{a}=\xi u^{a}$ is a well-known equation $£_{\xi} g_{a b}=2 \psi g_{a b}$, which is equivalent to the following conditions [21]

$$
\begin{aligned}
\dot{u}_{a}-(\ln \xi)_{; a} & =u_{a}(\ln \xi)^{\cdot}=\xi^{-1} \psi u_{a} \\
\theta & =3 \xi^{-1} \psi \\
\sigma_{c d} & =0
\end{aligned}
$$

Proof: The symmetry equation of $\mathrm{CKV}$ is

$$
£_{\xi} g_{a b}=2 \psi g_{a b}
$$

which implies that

$$
\xi_{a ; b}+\xi_{b ; a}=2 \psi g_{a b} .
$$

Contracting the above equation in turn with $u^{a} u^{b}, u^{a} h_{c}^{b}, h^{a b}$ and $h_{c}^{a} h_{d}^{b}-\frac{1}{3} h^{a b} h_{c d}$ and applying $\xi^{a}=\xi u^{a}$, we have

$$
\begin{aligned}
u_{a}(\ln \xi) & =\xi^{-1} \psi, \\
\dot{u}_{a}-(\ln \xi)_{; a}-(\ln \xi) \cdot u_{a} & =0, \\
\theta & =3 \xi^{-1} \psi, \\
\sigma_{c d} & =0 .
\end{aligned}
$$

(i) Making use of (A5) in (A4), we have the condition (A1).

(ii) Eqs.(A6) and (A7) directly imply the conditions (A2) and (A3).

By putting these conditions in $\xi_{a ; b}+\xi_{b ; a}$, where $\xi^{a}=\xi u^{a}$, we obtain $2 \psi g_{a b}$ and so the conditions are necessary as well as sufficient.

When $\psi=0$, the conditions (A1)-(A3) reduces to the necessary and sufficient condition for the existence of timelike $\mathrm{KV}$. 


\section{Appendix B}

\section{Conditions for Spacelike CKVs}

Here we prove the necessary and sufficient conditions for a SpCKV.

Theorem: The primary effect of a $\operatorname{SpCKV} \xi^{a}=\xi x^{a}$ is a well-known equation $£_{\xi} g_{a b}=2 \psi g_{a b}$, which is equivalent to the following conditions [8]

$$
\begin{aligned}
S_{a b} & =0, \\
x_{a}^{*}+(\ln \xi)_{, a} & =\frac{1}{2} \theta^{*} x_{a}, \\
u_{a} \dot{x}^{a} & =-\frac{1}{2} \theta^{*}, \\
N_{a} & =-2 \omega_{a b} x^{b}, \\
\psi & =\frac{1}{2} \xi \theta^{*}=\xi^{*} .
\end{aligned}
$$

Proof: The symmetry equation of $\mathrm{CKV}$ is

$$
£_{\xi} g_{a b}=2 \psi g_{a b}
$$

which implies that

$$
\xi_{a ; b}+\xi_{b ; a}=2 \psi g_{a b} .
$$

Contracting the above equation in turn with $u^{a} u^{b}, u^{a} x^{b}, u^{a} H^{b c}, x^{a} x^{b}, x^{a} H^{b c}$ and $H^{a c} H^{b d}$ and applying $\xi^{a}=\xi x^{a}$, we have

$$
\begin{aligned}
u_{a} \dot{x}^{a} & =-\frac{\psi}{\xi}, \\
u^{a}\left[x_{a}^{*}+(\ln \xi)_{, a}\right] & =0, \\
H^{a b}\left[\dot{x}_{b}+u^{t} x_{t ; b}\right] & =0, \\
\xi^{*} & =\psi, \\
H^{a b}\left[x_{b}^{*}+(\ln \xi)_{, b}\right] & =0, \\
S_{a b}+\frac{1}{2}\left[\theta^{*}-\frac{2 \psi}{\xi}\right] H_{a b} & =0 .
\end{aligned}
$$

(i) Multiply (B11) by $g^{a b}$, we have

$$
S_{a}^{a}+\frac{1}{2}\left[\theta^{*}-\frac{2 \psi}{\xi}\right] H_{a}^{a}=0 .
$$


This implies that $\theta^{*}=\frac{2 \psi}{\xi}$. This equation with Eq.(B9) gives condition (B5).

(ii) Replacing the value of $\psi$ from Eq.(B5) in Eq.(B6), we have condition (B3).

(iii) Eqs.(B7) and (B10) implies that

$$
x_{a}^{*}+(\ln \xi)_{; a}-x_{a}(\ln \xi)^{*}=0 .
$$

As

$$
(\ln \xi)^{*}=\frac{\xi^{*}}{\xi}=\frac{\theta^{*}}{2}
$$

the above equation gives

$$
x_{a}^{*}+(\ln \xi)_{, a}-\frac{\theta^{*} x_{a}}{2}=0
$$

or

$$
x_{a}^{*}+(\ln \xi)_{, a}=\frac{1}{2} \theta^{*} x_{a}
$$

which is condition (B2).

(iv) Substituting the value of $\psi$ from Eq.(B5) in Eq.(B11), Eq.(B1) turns out.

(v) On expanding Eq.(B8), we have

$$
H_{a}^{b}\left(\dot{x_{b}}+u_{b}^{*}\right)+2 \omega_{a b} x^{b}=0
$$

which gives condition (B4).

Substituting these conditions in $\xi_{a ; b}+\xi_{b ; a}$ where $\xi^{a}=\xi x^{a}$, we obtain $2 \psi g_{a b}$ and so the conditions are necessary as well as sufficient. When $\psi=0$, the conditions (B1)-(B5) reduce to the necessary and sufficient conditions of SpKVs. 


\section{References}

[1] Duggal, K.L.: J. Math. Phys. 33(1992)2989.

[2] Herrera, L. and Ponce de, L.J.: J. Math. Phys. 26(1985)778; 26(1985)2018; 26(1985)2847.

[3] Maartens, R., Mason, D.P. and Tsamparlis, M.: J. Math. Phys. 27(1986)2987.

[4] Coley, A.A. and Tupper, B.O.J.: J. Math. Phys. 30(1989)2616.

[5] Carot, J., Coley, A.A., and Sintes, A.: Gen. Rel. Grav. 28(1986)311.

[6] Duggal, K.L.: Acta Appl. Math. 31(1993)225.

[7] Greenberg, P.J.: J. Math. Anal. Appl. 30(1970)128.

[8] Mason, D.P. and Tsamparlis, M.: J. Math. Phys. 24(1983)1577.

[9] Yavuz, $\dot{I}$. and Yilmaz, $\dot{I} .:$ Gen. Rel. Grav. 29(1997)1295.

[10] Yilmaz, $\dot{I}$. ., Tarhan, $\dot{I}$., Yavuz, $\dot{I}$. , Baysal, H. and Camci, U.: Int. J. Mod. Phys. D8(1999)659.

[11] Baysal, H., Camci, U., Yilmaz, $\dot{I}$. and Tarhan, $\dot{I} .:$ Int. J. Mod. Phys. D11(2002)463.

[12] Yilmaz, I.: Int. J. Mod. Phys. D10(2001)681.

[13] Baysal, H. and Yilmaz, İ: Class. Quantum Grav. 19 (2002)6435.

[14] Baysal, H. and Yilmaz, I: Turk J. Phys 27(2003)83.

[15] Zeldovich, Ya B.: Mon. Not. R. Astron. Soc. 192(1980)663.

[16] Kibble, T.W.S.: J. Phys. A: Math. Gen. 9(1976)1387.

[17] Letelier, Patricio S.: Phys. Rev. D22(1980)807.

[18] Letelier, Patricio S.: Phys. Rev. D28(1983)2414.

[19] Letelier, Patricio S.: Nuovo Cimento 63B(1981)519. 
[20] Stephani, Hans: General Relativity: An Introduction to the Theory of the Gravitational Field (Cambridge University Press, 1990).

[21] Oliver, D.R. and Davis, W.R.: Gen. Rel. Grav. 8(1977)905.

[22] Duggal, K.L. and Sharma, R.: Symmetries of Spacetimes and Riemannian Manifolds (Kluwer Academic Publishers, 1999). 